\title{
High-frequency oscillations in a solar active region observed with the RAPID DUAL IMAGER
}

\author{
D. B. Jess ${ }^{1,2}$, A. Andić ${ }^{1}$, M. Mathioudakis ${ }^{1}$, D. S. Bloomfield ${ }^{3}$, and F. P. Keenan ${ }^{1}$
}

\author{
1 Astrophysics Research Centre, School of Mathematics and Physics, Queen's University, Belfast, BT7 1NN, Northern Ireland, UK \\ e-mail: djess01@qub.ac.uk \\ 2 NASA Goddard Space Flight Center, Solar Physics Laboratory, Code 612.1, Greenbelt, MD 20771, USA \\ ${ }^{3}$ Max-Planck-Institut für Sonnensystemforschung, Max-Planck-Str. 2, 37191 Katlenburg-Lindau, Germany
}

Received 22 January 2007 / Accepted 16 July 2007

\begin{abstract}
High-cadence, synchronized, multiwavelength optical observations of a solar active region (NOAA 10794) are presented. The data were obtained with the Dunn Solar Telescope at the National Solar Observatory/Sacramento Peak using a newly developed camera system: the RAPID DUAL IMAGER. Wavelet analysis is undertaken to search for intensity related oscillatory signatures, and periodicities ranging from 20 to $370 \mathrm{~s}$ are found with significance levels exceeding $95 \%$. Observations in the $\mathrm{H}-\alpha$ blue wing show more penumbral oscillatory phenomena when compared to simultaneous G-band observations. The H- $\alpha$ oscillations are interpreted as the signatures of plasma motions with a mean velocity of $20 \mathrm{~km} \mathrm{~s}^{-1}$. The strong oscillatory power over H- $\alpha$ blue-wing and G-band penumbral bright grains is an indication of the Evershed flow with frequencies higher than previously reported.
\end{abstract}

Key words. instrumentation: miscellaneous - waves - Sun: chromosphere - Sun: oscillations - Sun: photosphere - Sun: sunspots

\section{Introduction}

Since the discovery of solar oscillations in the 1960s (Leighton 1960), and their subsequent confirmation by Deubner (1975), there has been a multitude of observational evidence presented verifying the existence of oscillations in the solar atmosphere (Stein \& Leibacher 1974). Oscillations have been suggested as candidates to explain one of the main unanswered questions in solar physics - Why is the outer solar atmosphere hotter than its surface? Acoustic oscillations as a mechanism to support atmospheric heating in the form of wave dissipation was postulated by Schwarzschild (1948) and Biermann (1948). Theory suggests that this form of magnetohydrodynamic (MHD) wave can either propagate upwards from the lower solar atmosphere or be induced in active regions by reconnection events (Hollweg 1981). Early work concerned with oscillatory phenomena in highly magnetic structures (e.g. sunspots), have validated the detection of low-frequency oscillations which are a response of the umbral photosphere to the 5-min p-mode global oscillations (see review by Lites 1992). Furthermore, Bloomfield et al. (2007) have examined wave modes within sunspot penumbra and have determined that modified p-mode waves exhibit the best agreement with current observations. Similarly, Marcu \& Ballai (2005) have studied oscillations with reference to the propagation of compressional MHD waves in penumbral filamentary structures in the photosphere.

Extensive analyses of low-frequency $(\leq 3.6 \mathrm{mHz})$ oscillations has shown evidence for mode coupling in the lower solar atmosphere (McAteer et al. 2003; Bloomfield et al. 2004). Higher-frequency acoustic oscillations $(3.3-33 \mathrm{mHz})$ have been detected in the chromosphere (White \& Athay 1979a,b; and Athay \& White 1978, 1979a,b), but it was found that the total energy flux of the oscillations was two orders of magnitude lower than that required to balance the radiative losses from the chromosphere. Further work on high-frequency oscillations has been performed by Williams et al. (2001, 2002), who interpret $6 \mathrm{~s}$ intensity oscillations as longitudinal magneto-acoustic waves in an active region coronal loop, and by Andić (2007) who investigated the radial component of chromospheric oscillations with periodicities as short as $45 \mathrm{~s}$.

Following analysis of TRACE data, Fossum \& Carlsson (2005a) were unable to detect sufficient power in high-frequency waves and concluded that these waves cannot constitute the dominant heating mechanism of the chromosphere. However, this study is limited by the cadence TRACE can achieve and the onboard filter transmissions (Fossum \& Carlsson 2005b). This means that physically small oscillation sites with short coherence lengths may be smeared out by the coarse sampling. In addition this method may overlook dynamic patterns created on spatial scales too small to be resolved with TRACE (Wedemeyer-Böhm et al. 2007; Jefferies et al. 2006).

Another interesting area incorporating oscillatory phenomena is the work concerned with Evershed flows (see Solanki 2003, for a review). Pioneering work by St. John (1913) states that the Evershed velocity flow in sunspots induces plasma behaviour which is detectable both in the photosphere and chromosphere. This flow of plasma is readily observed in $\mathrm{H}-\alpha$ wings and has an associated flow speed of up to $20 \mathrm{~km} \mathrm{~s}^{-1}$.

To date, there has been a multitude of observations undertaken to search for oscillations in the upper solar atmosphere (chromosphere, transition region and corona). However, very little work has been carried out to search for high-frequency oscillations in the photosphere, where the building blocks of wave generation and propagation may exist.

Van Noort \& Rouppe van der Voort (2006) have verified the existence of highly dynamic structures, including propagating waves with velocities exceeding $200 \mathrm{~km} \mathrm{~s}^{-1}$, in the chromosphere. This, coupled with the detection of fast fluctuations of 
$\mathrm{H}-\alpha$ emission, from a flare kernel on timescales of $0.3-0.7 \mathrm{~s}$, by Wang (2005) demonstrates the need for high-cadence solar imaging. Furthermore, using typical chromospheric plasma parameters, the cooling time of the chromosphere is approximately $1 \mathrm{~s}$ (Heinzel 1991). Therefore, in order to study such rapid atmospheric variations, it is necessary to implement high-cadence imaging techniques. When searching for rapid solar variability, in particular, high-frequency oscillations, there are many obstacles which first must be overcome. The search for rapid, often low-amplitude intensity variations requires a suitably high cadence to satisfy the Nyquist parameter, a highly sensitive camera system providing accurate, sustained frame rates, good seeing and a minimally weakened signal caused by the convolution of wave perturbations with a wide response function.

Here we report intensity oscillations detected within the lower solar atmosphere using a new camera system developed at Queen's University Belfast - the RAPID DUAL IMAGER (RDI). In Sect. 2 we provide a brief background to the RDI system used for our observations, which are described in detail in Sect. 3. In Sect. 4 we discuss the methodologies used during the analysis of the observations and the search for reliable oscillatory signatures. A discussion of our results in the context of Evershed oscillations and penumbral waves is in Sect. 5, and finally, our concluding remarks are given in Sect. 6.

\section{Rapid dual imager}

The RDI was developed as a follow on to the highly successful SECIS (Phillips et al. 2000) camera system and consists of two Basler A301b cameras - one "master" and one "slave" channel - connected to a custom built PC, and controlled by software designed and developed by 4C's of Somerset, UK. The cameras have a $502 \times 494$ pixel $^{2} \mathrm{CCD}$, with a square pixel size of $9.9 \mu \mathrm{m}$ and can operate at a maximum speed of $80 \mathrm{fps}$. Their lightweight, robust design and $\mathrm{C}$-mount housing make them ideal for a portable system such as RDI. The camera mounts act as passive heatsinks, ensuring that the cameras are kept within the recommended operating temperatures.

Each camera is controlled via a PCI interface card. A lossless compression algorithm loaded into an on-board fieldprogrammable gate array increases the data throughput rate to the PC. The cameras are attached to the control cards via 5-mlong high-bandwidth cables which carry data, command signals (via a standard RS-232 serial link) and power, and are synchronized via a ribbon connecting the interface cards. The master card's clock is set from the PCI bus clock; the slave clock is in turn synchronized to the master clock so that both channels are truly synchronous. RDI's high rate of data recording is made possible using an IDE RAID array of two pairs of "striped" disks, each pair acting as one data storage area, assigned to a particular camera. While images are streamed to two preallocated files, sample images from the stream are displayed on-screen to allow the observers to check factors such as image quality and seeing conditions.

The preallocated space is filled at a semi-compressed rate of $195 \mathrm{kB}$ per image per camera. For maximum window size at a frame rate of $20 \mathrm{~Hz}$, this corresponds to an uninterrupted data run of over $7 \mathrm{~h}$ using the full available $200 \mathrm{~GB}$ of hard-disk space. The data are then converted to FITS format using the built in conversion software and backed up onto tape drives or external hard-drives. RDI is equipped with a multitude of additional features, such as the ability to window specific, user-defined regions of the field of view and also to bin multiple groups of pixels together for increased count-rates. RDI remains stationed at the
Table 1. Details of image acquisition.

\begin{tabular}{lccc}
\hline \hline Observation & $\begin{array}{c}\text { Central } \\
\text { wavelength } \\
(\AA)\end{array}$ & $\begin{array}{c}\text { Filter } \\
\text { bandpass } \\
(\AA)\end{array}$ & $\begin{array}{c}\text { Height of } \\
\text { formation } \\
(\mathrm{km})\end{array}$ \\
\hline G-band & 4305.50 & 9.20 & $<250^{a, b}$ \\
H- $\alpha$ blue wing & 6561.51 & $0.21^{c}$ & $<200^{d, e}$ \\
\hline
\end{tabular}

${ }^{a}$ Uitenbroek \& Tritschler (2006). ${ }^{b}$ Rimmele (2004). ${ }^{c}$ Beckers et al. (1975). ${ }^{d}$ Ding et al. (2001). ${ }^{e}$ Leenaarts et al. (2006).

National Solar Observatory, Sacramento Peak as a common-user instrument.

\section{Observations}

The data presented here are part of an observing sequence obtained on 2005 August 10, with the Richard B. Dunn Solar Telescope (DST) at Sacramento Peak. The optical setup allowed us to image a $50.4^{\prime \prime} \times 49.2^{\prime \prime}$ region surrounding active region NOAA 10794 complete with solar rotation tracking. The active region under investigation was located at heliocentric co-ordinates $\left(770^{\prime \prime},-254^{\prime \prime}\right)$, or S12W56 in the solar NS-EW co-ordinate system. A Zeiss universal birefringent filter (UBF; Beckers et al. 1975) was used for $\mathrm{H}-\alpha$ blue-wing ( $\mathrm{H}-\alpha$ core $1.3 \AA$ ) imaging with one of the RDI CCD detectors. In addition, a G-band filter was employed with the second RDI camera to enable synchronized imaging in the two wavelengths. During the observations presented here, low-order adaptive optics was implemented.

The observations employed in the present analysis consist of 72000 images in each wavelength, taken with a 0.05 s cadence, providing one hour of uninterrupted data. The acquisition time for this observing sequence was early in the morning and seeing levels were good with minimal variation throughout the time series. Table 1 lists the wavelengths and filter passbands used in the image acquisition, alongside their approximate heights of formation. The height of formation of the $\mathrm{H}-\alpha$ line profile has long been a topic of key interest among ground-based solar observers. Leenaarts et al. (2005) have carried out magnetoconvection simulations and show that the formation height of the $\mathrm{H}-\alpha$ blue wing (core $-0.8 \AA$ ) is well under $500 \mathrm{~km}$. Thus, our use of the far $\mathrm{H}-\alpha$ blue wing will provide a formation height lower than this, forming at a height of $200 \mathrm{~km}$ or less (Leenaarts et al. 2006). The $\mathrm{H}-\alpha$ line, however, is very sensitive to solar activity. Indeed, during flares the formation height can be pushed into the chromosphere up to a height of approximately $1500 \mathrm{~km}$ (Ding et al. 2001). During the observations discussed here, solar activity was minimal with no flare activity registered within \pm 4 days. Utilizing the models of Leenaarts et al. (2005, 2006) we therefore conclude that the formation height of the H- $\alpha$ blue wing in our observations is less than $200 \mathrm{~km}$.

A comparison of Figs. 1 and 2 reveals that many solar features, (sunspot, bright points, granulation etc.), are co-spatial and of similar proportions, thus indicating that the H- $\alpha$ bluewing (core $-1.3 \AA$ ) forms at an atmospheric height similar to the G-band. Indeed, Uitenbroek \& Tritschler (2006), through the use of high-resolution synthetic $\mathrm{CH}$ - and $\mathrm{CN}$-band filtergrams, compute the formation height of the G-band to be approximately $200 \mathrm{~km}$. Several differences, however, between the two filter bandpasses exist. For example, magnetic elements in the $\mathrm{H}-\alpha$ blue wing appear to be much brighter and granulation contrast much weaker when compared to simultaneous G-band 

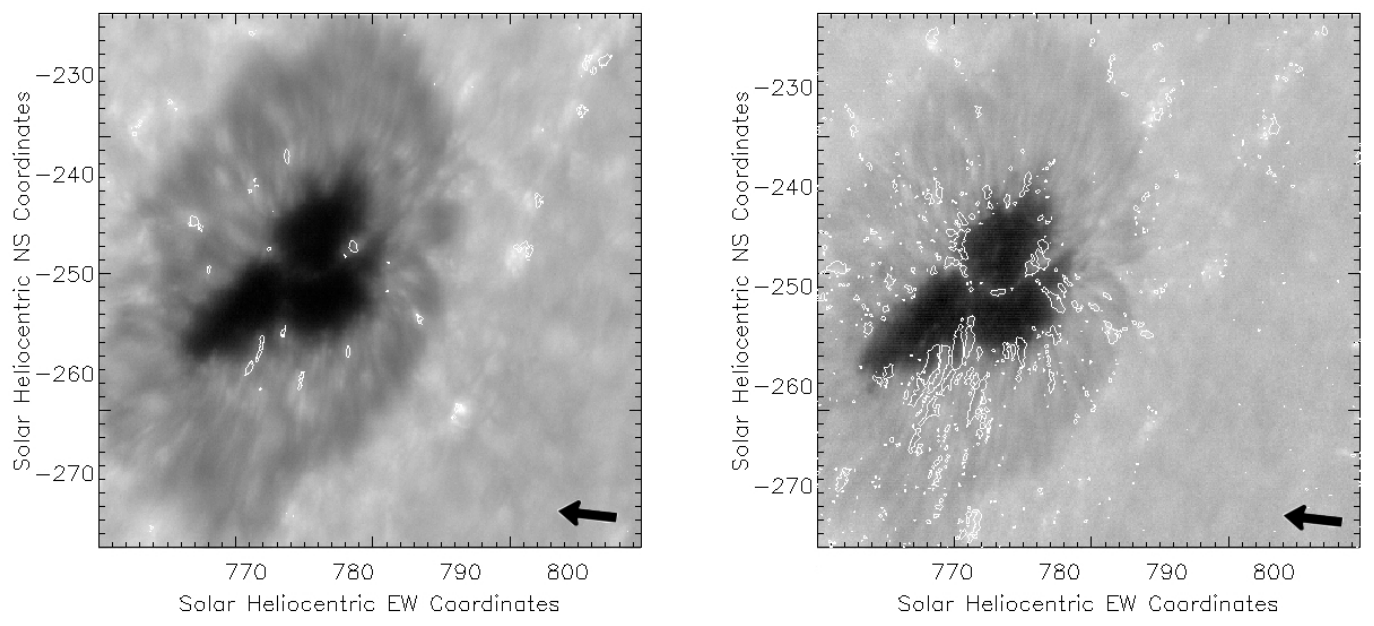

Fig. 1. The G-band (left) and H- $\alpha$ blue-wing (right) field of view. The white contours mark areas where oscillations with a $26 \mathrm{~s}$ periodicity are detected for a particular instant in time. The scale is in heliocentric co-ordinates $\left(1\right.$ pixel $\left.=0.1^{\prime \prime}\right)$ and all contoured oscillation sites have confidence levels above $99 \%$ and abide by the criteria enforced in Sect. 4.1. The arrow indicates the direction of disc centre.
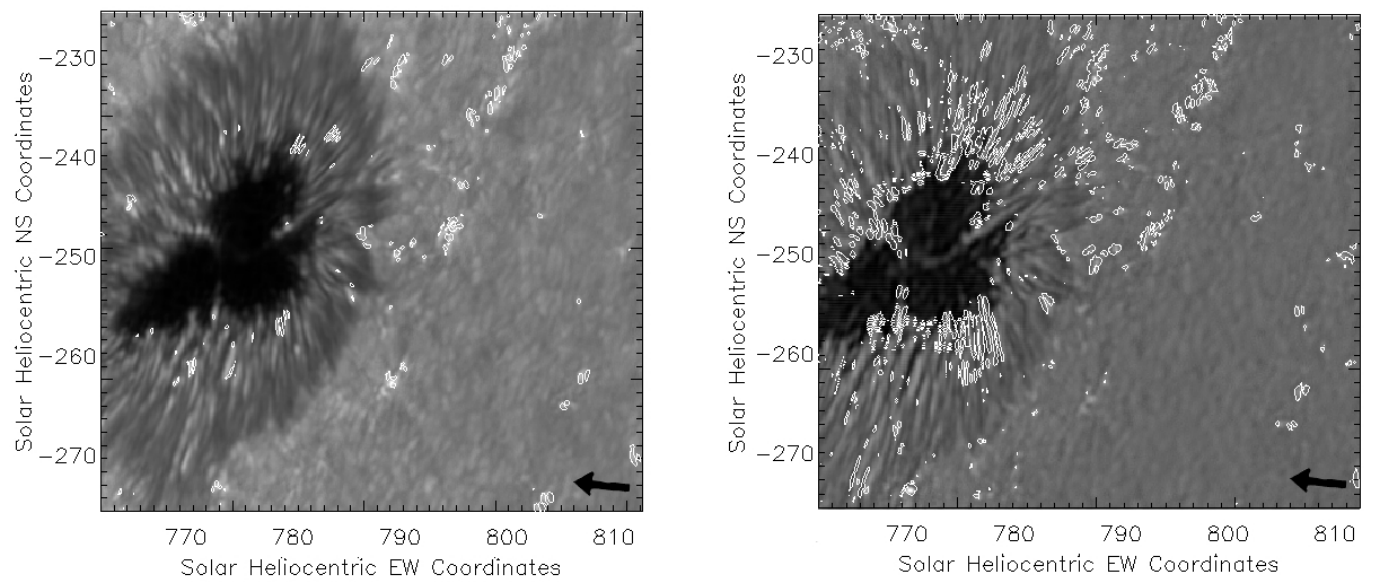

Fig. 2. Speckle reconstructed G-band (left) and H- $\alpha$ blue-wing (right) images. The white contours mark areas where oscillations with a $33 \mathrm{~s}$ periodicity are detected. The scale is in heliocentric co-ordinates $\left(1\right.$ pixel $\left.=0.1^{\prime \prime}\right)$ and all contoured oscillation sites have confidence levels above $99 \%$ and abide by the criteria enforced in Sect. 4.1. The arrow indicates the direction of disc centre.

images. Thus, the similar height formation of the two chosen bandpasses, added to their subtle imaging differences, provide us with an interesting platform to study processes occurring in the photosphere. The acquired images have a sampling of $0.1^{\prime \prime}$ per pixel to match the telescope's diffraction limited resolution in the $\mathrm{H}-\alpha$ blue wing to that of the CCD. The optical path to both cameras were identical meaning the G-band camera was slightly undersampled. This is desirable to keep the dimensions of the field of view the same for both cameras.

\section{Data analysis}

\subsection{Initial image processing}

After implementing temporal Fourier analysis on 2400 successive dark images, a moving-pattern noise was revealed, showing up as an intense Fourier power with a variable periodicity between 3 and 9 s. This banding was identical in magnitude and periodicity on both cameras. To insure this camera induced moving-pattern noise did not interfere with the analysis, all periodicities less than $20 \mathrm{~s}$ were neglected. Arrays of dark-subtracted and flat-fielded data were saved for each camera for subsequent analysis. To compensate for camera jitter and large-scale airpocket motions, all data was subjected to a Fourier co-aligning routine commonly available in the SSW tree of IDL. This routine utilizes cross-correlation techniques as well as squared mean absolute deviations to provide sub-pixel co-alignment accuracy. After 5 successive co-alignments of the data, the maximum $x$ and $y$-displacements, over the entire duration of the dataset, are both less than one tenth of a pixel. Since the observing sequence was obtained in the early hours of the morning, when image warping is particularly strong, all data were de-stretched relative to simultaneous, high-contrast G-band images. We use a $40 \times$ 40 grid, equating to a $1.25^{\prime \prime}$ separation between spatial samples, to evaluate local offsets between successive G-band images. Due to both cameras sharing the same pre-filter optical path, all determined local offsets are applied to simultaneous narrowband images to compensate for spatial distortions caused by atmospheric turbulence and/or air bubbles crossing the entrance aperture of the telescope. The fine destretching grid implemented in this process allows small-scale seeing conditions, of $1^{\prime \prime}$ to $2^{\prime \prime}$, to be compensated for.

After successful co-alignment and destretching, lightcurves were created for each pixel of each camera before being passed into Fast Fourier Transform (FFT) and wavelet analysis routines. While a FFT searches for periodic signatures by decomposing the input signal into infinite length sinusoidal wavetrains using a 
basic exponential function, wavelet analysis utilizes a time localised oscillatory function continuous in both frequency and time (Bloomfield et al. 2004) and is therefore highly suited in the search for transient oscillations. The wavelet chosen for this study is known as a Morlet wavelet and is the modulation of a sinusoid by a Gaussian envelope (Torrence \& Compo 1998). Strict criteria were implemented during wavelet analysis to insure that oscillatory signatures correspond to real periodicities. The first is a test against spurious detections of power that may be due to Poisson noise, where the input lightcurve is assumed to be normally distributed (consistent with photon noise) and following a $\chi^{2}$ distribution with two degrees of freedom. A $99 \%$ confidence level is calculated by multiplying the power in the background spectrum by the values of $\chi^{2}$ corresponding to the 99 th percentile of the distribution (Torrence \& Compo 1998; Mathioudakis et al. 2003).

The second criterion applied is a comparison of the input lightcurve with a large number (1500) of randomized time-series with an identical distribution of counts. The probability, $p$, of detecting non-periodic power is calculated for the peak power at each timestep by comparing the value of power found in the input lightcurve with the number of times that the power transform of the randomized series produces a peak of equal or greater power. A percentage confidence is attributed to the peak power at every time step in the wavelet transform by $(1-p) \times 100$, such that a high value of $p$ implies that there is no periodic signal in the data, while a low value suggests that the detected periodicity is real (see Banerjee et al. 2001).

Our final wavelet criterion is the exclusion of oscillations which last, in duration, less than 1.41 cycles. This is consistent with the decorrelation time defined by Torrence \& Compo (1998). One can distinguish between a spike in the data and a harmonious periodic component at the equivalent Fourier frequency by comparing the width of a peak in the wavelet power spectrum with the decorrelation time. From this, the oscillation lifetime at the period of each power maximum is defined as the interval of time from when the power supersedes $95 \%$ significance to when it subsequently dips below $95 \%$ significance (McAteer et al. 2004). The lifetime was then divided by the period to give a lifetime in terms of complete cycles (Ireland et al. 1999). Any oscillations which last for less than this minimum duration were discarded as they may have simply been a spike in the lightcurve.

Four-dimensional maps containing spatial information as well as wavelet power and oscillatory period were saved as outputs of wavelet analysis for detected oscillations which lay above the $95 \%$ significance level set by the criteria above.

\subsection{Speckle reconstructed data}

Small-scale turbulent seeing in the Earth's atmosphere means that even high-order adaptive optics cannot compensate for all rapid air motions, and speckle reconstruction is often used as a powerful post-processing routine designed to restore the data to diffraction-limited resolution. Here we implement the speckle masking method of Weigelt \& Wirnitzer (1983), adapted for solar imaging by von der Lühe (1993) and further improved by de Boer (1995). By observing at a high cadence, the short exposure times acquired essentially freeze out atmospheric distortions and maintain signals at high spatial frequencies, albeit with statistically disturbed phases (Sütterlin et al. 2001). It is possible to recover the true amplitudes and phases in Fourier space by taking a large number of such short exposure images, called a "Speckle Burst", and utilizing an elaborate statistical model.
Eighty raw data frames were used for each speckle reconstruction producing a new effective cadence of $4 \mathrm{~s}$. This provides a Nyquist frequency of $125 \mathrm{mHz}$ and is therefore suitable for the search of oscillations with periods longer than $8 \mathrm{~s}$. Typical Fried parameters obtained prior to speckle reconstruction were $r_{0} \approx 10 \mathrm{~cm}$, indicating good post-speckle image quality. However, to strengthen the reliability of our detections and to remain consistent with the analysis performed in Sect. 4.1, only oscillations with periodicities greater than $20 \mathrm{~s}$ were incorporated into the analysis. The above mentioned dark subtraction was performed identically prior to Speckle reconstruction, after which co-aligning and pixel-by-pixel lightcurve analysis was again implemented. Due to the increased spatial resolution provided by the implementation of Speckle reconstruction, only 3 successive runs of the co-alignment software was required to provide sub-pixel shifts. Again, four-dimensional maps containing spatial information as well as wavelet power and oscillatory period were saved as outputs of wavelet analysis for detected oscillations which lay above the $95 \%$ significance level.

\section{Results and discussion}

Both processed and speckle reconstructed data reveal signatures of high-frequency oscillations in the G-band and $\mathrm{H}-\alpha$ blue wing (Fig. 1). The well-established 5 min global oscillation can be seen predominantly in regions away from the sunspot (i.e. quiet sun), and indeed the 3 min umbral oscillation can be detected. This is consistent with the work of Brynildsen et al. (2002) who report oscillations with a period of $5 \mathrm{~min}$ are observed in sunspot umbrae, but with considerably less power than locations away from the sunspot. However, the oscillations we will concentrate on are those of much higher frequency. We have detected highFourier power at periodicities between 20 and $370 \mathrm{~s}$ in both the G-band and $\mathrm{H}-\alpha$ blue wing, with a significant concentration of high-frequency ( $>20 \mathrm{mHz}$ ) activity in the sunspot penumbra (Fig. 2). The detected oscillations have highly-correlated global wavelet power and Fourier power with the additional support of significance levels over 95\%. Figure 3 shows the detection of a $35 \mathrm{~s}$ oscillation originating from a dark penumbral filament within images acquired in the $\mathrm{H}-\alpha$ blue wing.

Figures 4 and 5 show histograms relating to the number of detected oscillations found, in the speckle reconstructed data, using the pixel by pixel wavelet analysis technique outlined in Sect. 4.1. It is clear that a large number of oscillations have been detected, and that the occurrence of higher frequency oscillations is greater in the $\mathrm{H}-\alpha$ blue wing. To further test the integrity of our results we binned $9(3 \times 3)$ spatial pixels together and recomputed the behaviour of oscillatory phenomena for both the G-band and H- $\alpha$ blue-wing data. This more coarse sampling will help prevent small-scale pixel noise below 3 pixels in amplitude from registering as Fourier power during wavelet analysis. From Fig. 6, it is clear that the results discussed above are unchanged even with coarse sampling.

In this work, we detect significant amounts of oscillatory power originating from bright penumbral grains (Figs. 7 and 8). We propose that this power, detected in both G-band and $\mathrm{H}$-alpha blue-wing observations, is closely linked to Evershed flow. A mechanism promoting Evershed flow was proposed by Schlichenmaier et al. (1998), who modelled the dynamic evolution of a thin flux tube inside the penumbra. A flux tube initially positioned at the magnetopause becomes buoyant due to radiative heating and rises. Pressure differences within the loop are created due to radiative cooling at the photosphere which drives an outward flow along the flux tube as it rises through the 

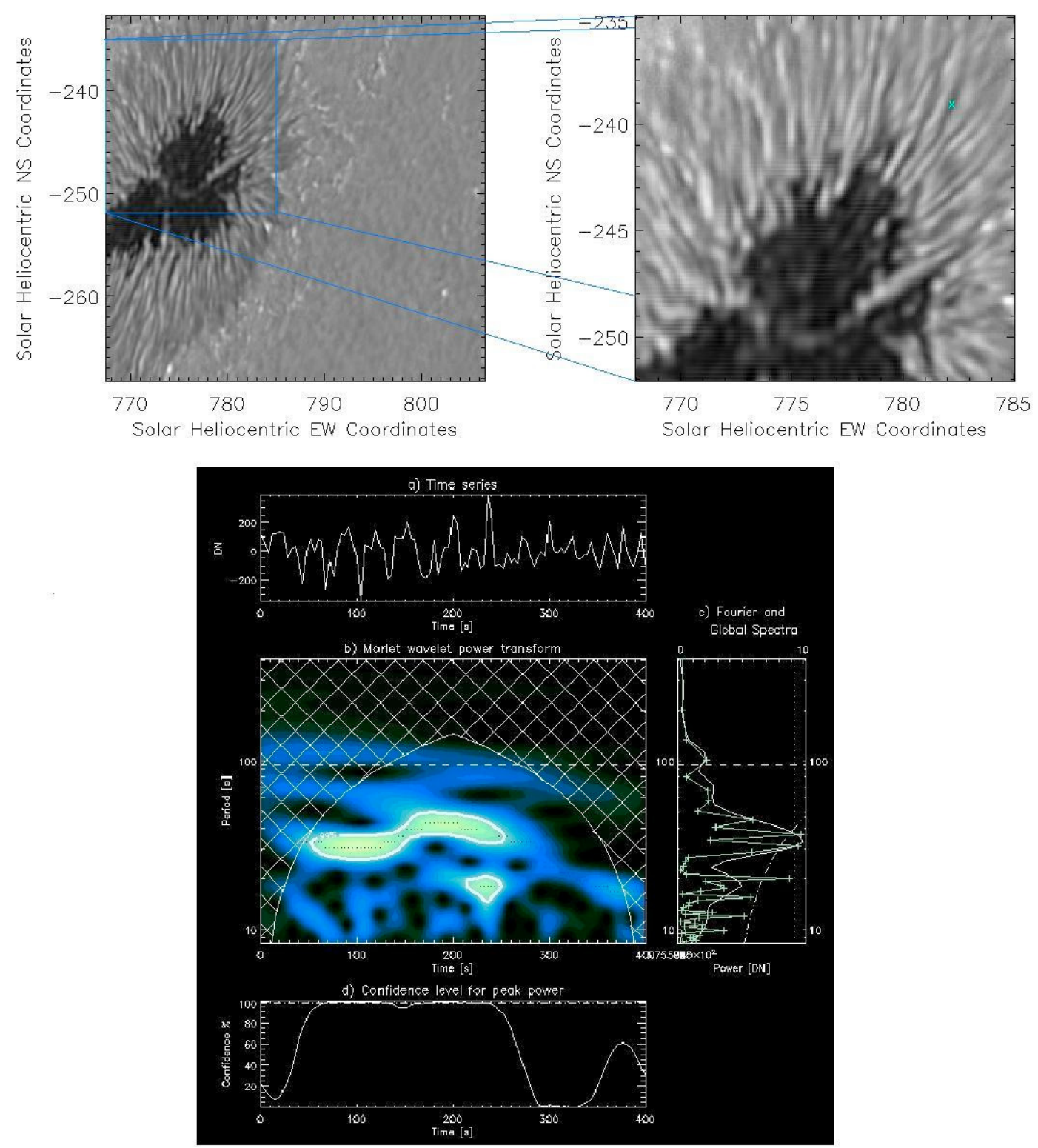

Fig. 3. The top left shows a frame taken from the $\mathrm{H}-\alpha$ blue-wing datacube followed by a zoomed in portion of the penumbra in the top right. The pixel location from which the lightcurve used in wavelet analysis was created is indicated by the cross. The bottom diagram shows the original pixel lightcurve in a). The wavelet power transform along with locations where detected power is at, or above, the $99 \%$ confidence level are contained within the contours in b). Plot c) shows the summation of the wavelet power transform over time (full line) and the Fast Fourier power spectrum (crosses) over time, plotted as a function of period. Both methods have detected a well pronounced $35 \mathrm{~s}$ oscillation. The global wavelet (dotted line) and Fourier (dashed dotted line) 95\% significance levels are also plotted. The cone of influence (COI), cross-hatched area in the plot, defines an area in the wavelet diagram where edge effects become important and as such any frequencies outside the COI are disregarded. Periods above the horizontal line (dotted) fall within the COI. The probability levels $(1-p) \times 100$ as discussed in Sect. 4.1 are plotted in d).

penumbra (Schlichenmaier et al. 1998). This model also predicts a filamentary structure characterized by a hot, nearly vertical upflow of plasma at the footpoint of the filament, which within several $100 \mathrm{~km}$ turns into a horizontal filament. We propose that the oscillations detected over bright penumbral grains correspond to the Evershed plasma flow along flux tubes anchored in the photosphere. Indeed, Schlichenmaier et al. (1998) state that footpoints of flux tubes creating Evershed flow within the penumbra could be identified by bright penumbral grains which reiterates our belief that such bright penumbral grain oscillations are associated with the Evershed flow.

In order to quantify our results, we determine the percentage of penumbral bright grains, both in the G-band and in the $\mathrm{H}-\alpha$ blue wing, which demonstrate oscillatory phenomena over a range of periodicities. By imposing a minimum bright grain intensity threshold (G-band: penumbral median $+5 \sigma, \mathrm{H}-\alpha$ blue wing: penumbral median $+8 \sigma$ ) we were able to mask out all regions excluding penumbral bright grains. By overplotting cotemporal locations of oscillations in the period range 28-33 s, we were able to determine the percentage of bright grains undergoing oscillatory phenomena. After examination of the entire time series, the average number of penumbral bright grains found in the G-band and $\mathrm{H}-\alpha$ blue wing, respectively, were 17 and 21 , using the minimum intensity threshold values stated above. With comparison to the location of high-frequency oscillatory power, we determine that $70 \%$ of the G-band bright grains exhibit co-spatial oscillations, while $79 \%$ of the $\mathrm{H}-\alpha$ bluewing bright grains demonstrate oscillatory perturbations. This 


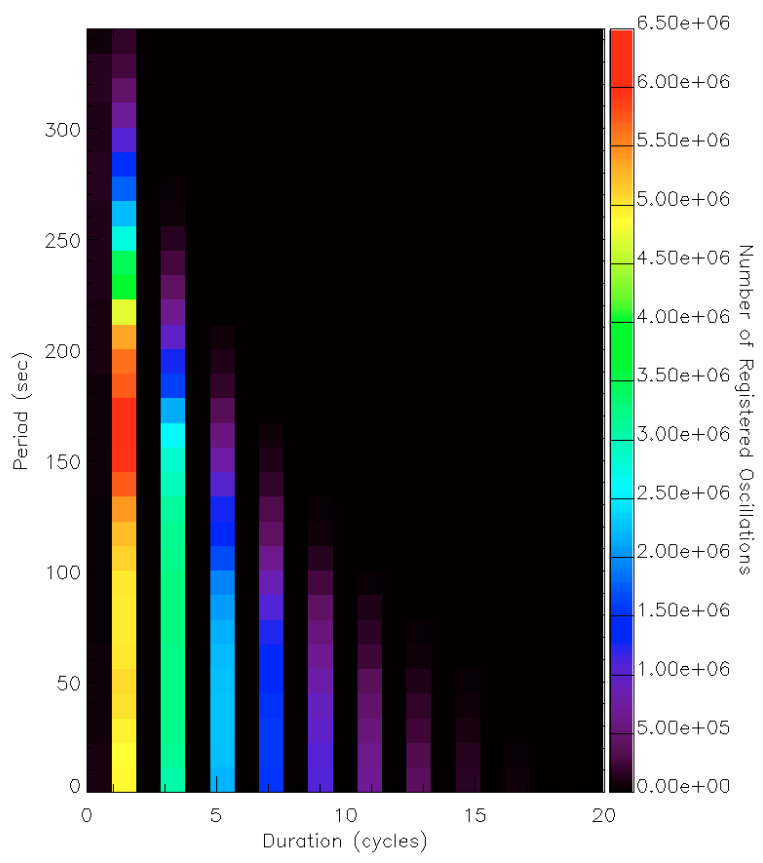

Fig. 4. The duration of oscillations is shown as a function of the period and this plot demonstrates the high occurrence of $\mathrm{H}-\alpha$ blue-wing oscillatory phenomena. The minimum oscillatory duration plotted is 1.41 cycles as described in Sect. 4.1. Each detected period is placed into bins corresponding to its cycle duration. For example, a lifetime of 2.65 cycles is placed in the $2-3.99$ cycle bin. Cycle duration bins are placed along the $x$-axis beginning with 1.41-1.99, 2-3.99, 4-5.99, 6-7.99 etc., while the detected period range is placed along the $y$-axis. Evaluated periods are again placed into bins which are $10 \mathrm{~s}$ in size beginning with a $0-9.99 \mathrm{~s}$ period bin. All oscillations with periodicities less than $20 \mathrm{~s}$ were not studied in order to fully satisfy the data's Nyquist parameter stated in Sect. 4.2. The colour scale represents the number of pixels detecting oscillatory phenomena throughout the entire time series and over the whole field of view which are consistent with the criteria enforced in Sect. 4.1.

reiterates our belief that the oscillations detected in penumbral bright grains are indicative of the Evershed flow, as described by Schlichenmaier et al. (1998).

From Figs. 1, 2 and 6 it is clear that we detect more oscillatory signatures in the $\mathrm{H}-\alpha$ blue-wing penumbra compared to the G-band penumbra. Since the formation heights of the $\mathrm{H}-\alpha$ blue wing and G-band are similar, we are unable to directly interpret these findings based solely on magneto-acoustic oscillations. Instead, we believe that this phenomena is a physical signature of velocity oscillations in the penumbra, whereby the $\mathrm{H}-\alpha$ line profile is periodically shifted due to plasma velocity flows. In this regime, wavelet analysis will detect the resulting periodic deviation in intensity as the $\mathrm{H}-\alpha$ line profile is moved across the filter's $0.21 \AA$ bandpass. Contrarily, the G-band samples continuum wavelengths and has a broad bandpass (9.2 $\AA$ ) and is therefore only sensitive to intensity rather than velocity variations. After investigating the amplitude of intensity variations in the $\mathrm{H}-\alpha$ blue-wing penumbra, we find that the average intensity variation detected during oscillations, with respect to an average quiescent value for that location on the penumbra, is approximately $11 \%$. Knowing the tuned wavelength of the blue-wing in the $\mathrm{H}-\alpha$ profile $(6561.51 \AA)$, it is possible to derive the necessary wavelength shift required to vary the intensity profile by the $11 \%$ observed here. Consulting a solar spectral atlas obtained using the Fourier Transform Spectrometer at the McMath/Pierce Solar Telescope, Kitt Peak, Arizona, we have

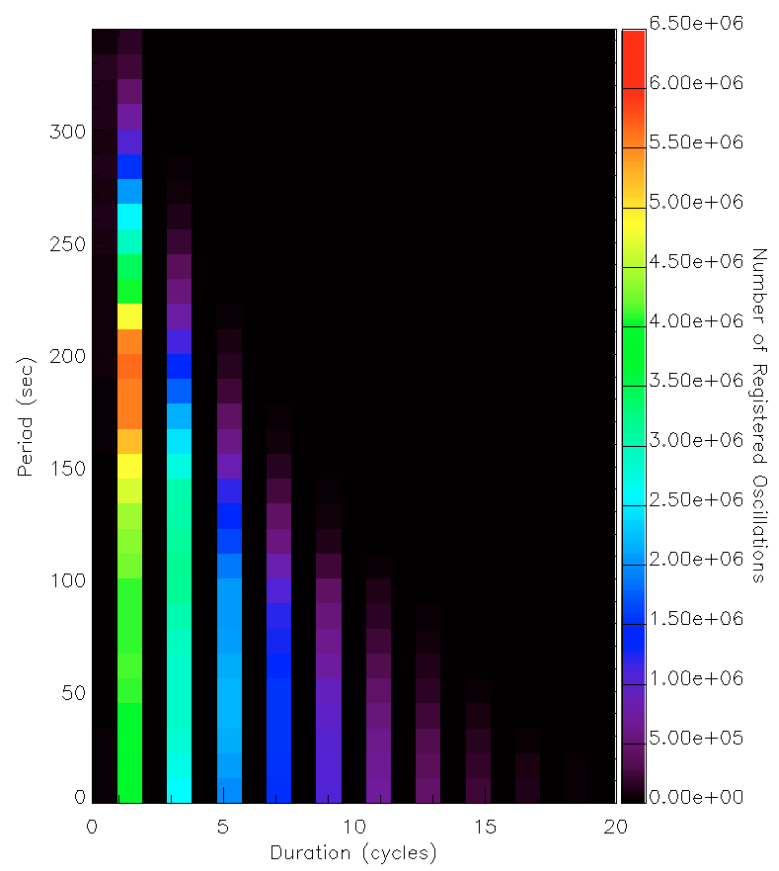

Fig. 5. Same as Fig. 4, but for the G-band. Notice the reduction in detected oscillations at higher frequencies when compared to Fig. 4. This can also be viewed via the spatial representation in Figs. 1, 2 and 6.

established the corresponding wavelength shift required to produce $11 \%$ amplitude oscillations is approximately $0.45 \AA$. This wavelength shift produces an estimate of the velocity associated with such plasma flows, and we derive this velocity to be $20 \mathrm{~km} \mathrm{~s}^{-1}$. Figure 9 shows the plasma velocities associated with the penumbra as observed in the $\mathrm{H}-\alpha$ blue wing. It must be noted, however, that since we are performing velocity analysis with narrow-band photometry rather than high-resolution spectroscopy, there will be errors in velocity associated with the width of the filter's bandpass. With the $0.21 \AA$ bandpass of the $\mathrm{UBF}$, this equates to velocity errors of the order of $\pm 5 \mathrm{~km} \mathrm{~s}^{-1}$. The use of a solar spectral atlas, for determining wavelength shifts from percentage intensity perturbations, will introduce some additional errors in the estimated velocities. The spectral atlas chosen is produced from disc-centre, quiet sun observations, and as such may differ if near-limb, active region data is used. Indeed, an $11 \%$ intensity amplitude could result in very different velocities between disc-centre and near-limb observations. Thus, it is imperative to stress potential errors associated with this form of analysis.

It has been known for quite some time that propagating waves exist in the penumbra of active regions. Zirin \& Stein (1972) found evidence for running penumbral waves (RPWs) in the chromosphere and Giovanelli (1972) who, through the analysis of spectroscopic line profiles, discovered associated velocity flows with RPWs of $20 \mathrm{~km} \mathrm{~s}^{-1}$. To date, there has been many detections of penumbral velocity flows ranging from 10 to $70 \mathrm{~km} \mathrm{~s}^{-1}$ (Brisken \& Zirin 1997; Kobanov et al. 2006) and our detection of $20 \mathrm{~km} \mathrm{~s}^{-1}$ velocities is within this range. The photospheric Evershed flow is usually associated with velocities of approximately $5 \mathrm{~km} \mathrm{~s}^{-1}$ (Bellot Rubio et al. 2003) which is well below our $20 \mathrm{~km} \mathrm{~s}^{-1}$ values. However, Schlichenmaier et al. (2002) provide a model where photospheric Evershed flows may reach velocities up to $14 \mathrm{~km} \mathrm{~s}^{-1}$, which is comparable with our findings after consideration of the errors involved in our analysis.

Previous observations of Evershed oscillations have detected periods ranging from 8 to $40 \mathrm{~min}$ (Rimmele 2004; 

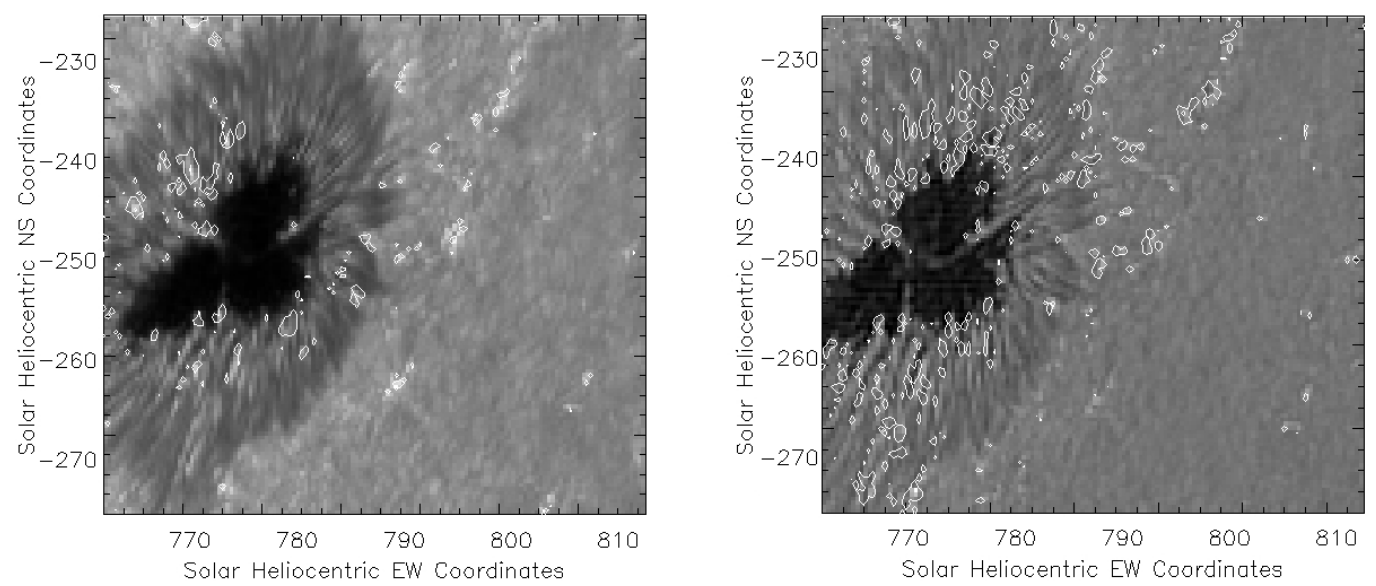

Fig. 6. The speckle reconstructed G-band (left) and H- $\alpha$ blue-wing (right) fields of view with $33 \mathrm{~s}$ oscillations overplotted. In both images the spatial sampling has been degraded by binning $9(3 \times 3)$ pixels together as discussed in Sect. 5 . It is clear to see that penumbral oscillations still dominate in the $\mathrm{H}-\alpha$ blue wing when compared to simultaneous G-band observations. The scale is in heliocentric co-ordinates and all contoured oscillation sites have confidence levels above $99 \%$ and abide by the criteria enforced in Sect. 4.1.

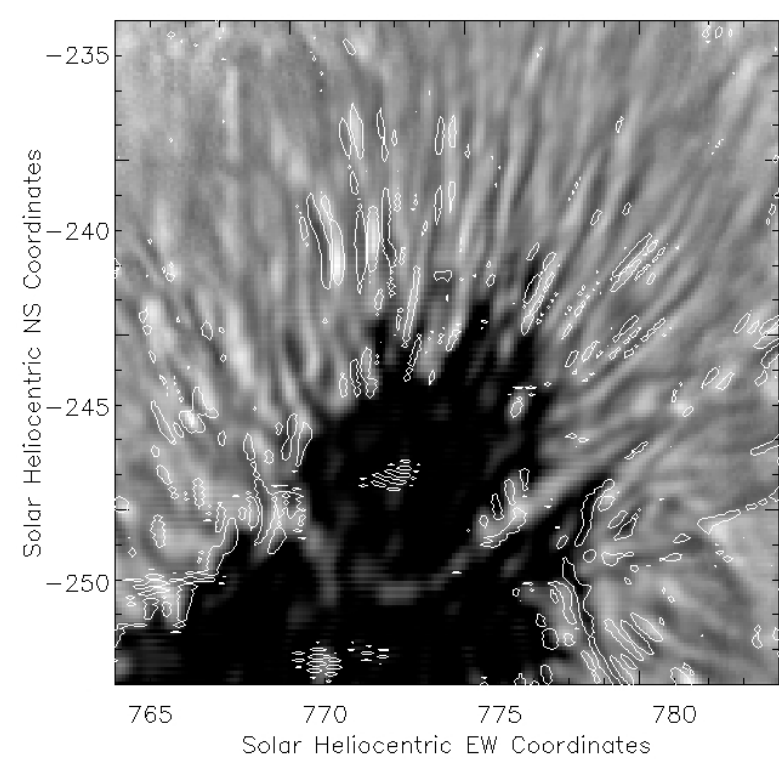

Fig. 7. Speckle reconstructed $\mathrm{H}-\alpha$ blue-wing image of the penumbral structure overplotted with locations of detected 33 s periodicity. The scale is in heliocentric co-ordinates and all contoured oscillation sites have confidence levels above $99 \%$ and abide by the criteria enforced in Sect. 4.1

Makarchik \& Kobanov 2001). We note that the penumbral bright-grain oscillations reported here are of much higher frequency than those observed to date. The importance of observations in the high-frequency domain, has been emphasized by Schlichenmaier \& Solanki (2003) who suggest that hot Evershed upflows may significantly contribute to the heating of the penumbra via the loss of energy through radiative processes as the plasma flows along the flux tubes away from the photosphere. In order to probe the viability of such heating mechanisms, it is necessary to combine high spatial and high temporal resolution Dopplergrams of non-magnetic lines (to provide a Doppler signal free from magnetic field effects) with highcadence vector-magnetograms. This will allow plasma flow velocities as well as surrounding magnetic field strength to be evaluated, which will enable derivations of the penumbral energy flux.

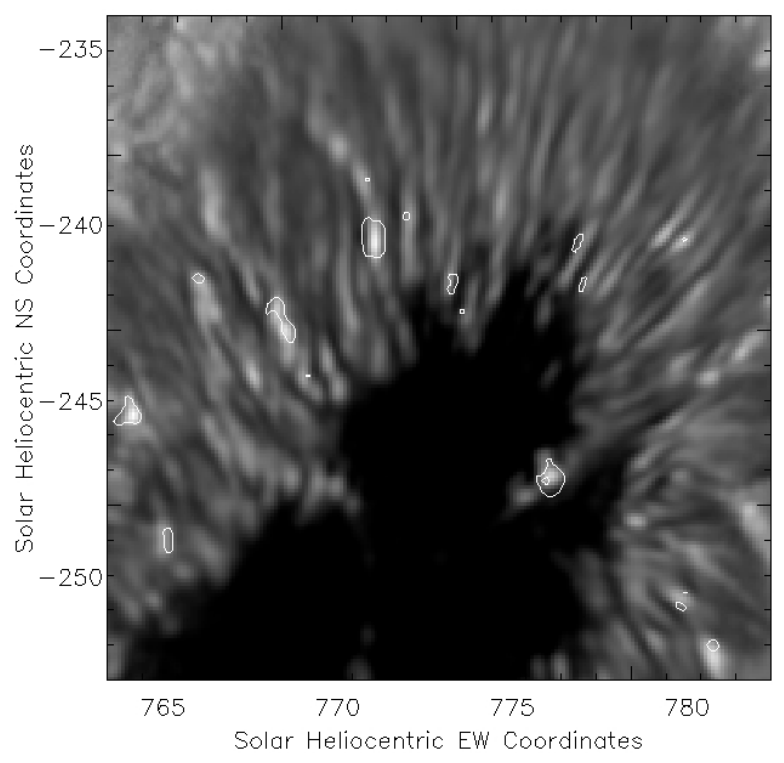

Fig. 8. Speckle reconstructed G-band image of the penumbral structure overplotted with the locations of $33 \mathrm{~s}$ oscillations. The scale is in heliocentric co-ordinates and all contoured oscillation sites have confidence levels above $99 \%$ and abide by the criteria enforced in Sect. 4.1. Note the reduction in oscillation sites compared with Fig. 7 and how such sites appear to be associated with bright penumbral grains.

\section{Concluding remarks}

We have presented direct evidence of high-frequency waves propagating in an active region, and detected oscillations with periodicities ranging from 20 to $370 \mathrm{~s}$ with significance levels greater than $95 \%$ due to the rigorous wavelet criteria enforced in Sect. 4.1. More penumbral oscillatory phenomena are found to be located in the H- $\alpha$ blue wing than in the G-band, and we conclude that these oscillations are associated with penumbral plasma flows.

$\mathrm{H}-\alpha$ blue-wing and G-band oscillations appear to have a strong spatial correspondence with penumbral bright grains. This promotes our belief that the detected high-frequency oscillations are associated with Evershed flows due to similarities with the model developed by Schlichenmaier et al. (1998). 


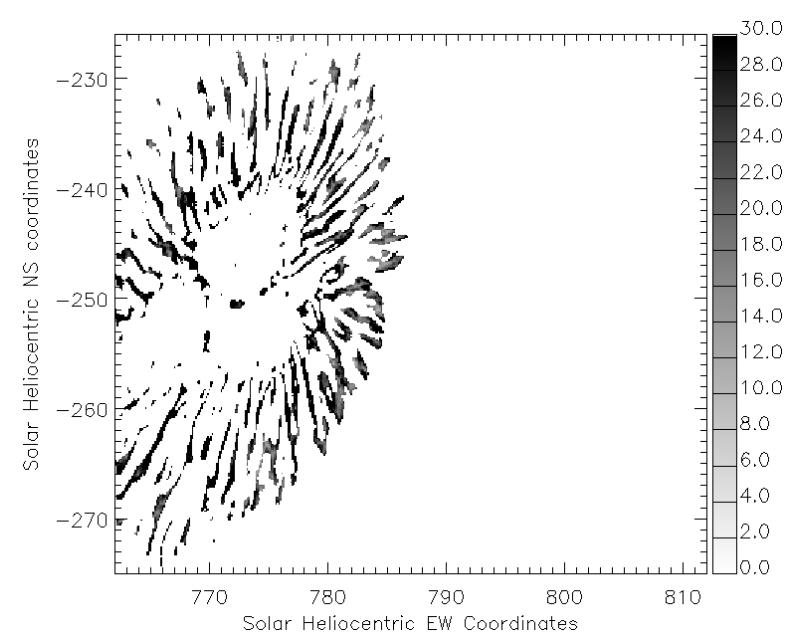

Fig. 9. Average velocity magnitudes of penumbral plasma flows in the $\mathrm{H}-\alpha$ blue wing determined from $28 \mathrm{~s}$ intensity oscillations. The velocities displayed represent those of the sunspot only, with all other areas masked out. The axis scales are in heliocentric co-ordinates while the colour scale provides an indication to the plasma flow velocity in $\mathrm{km} \mathrm{s}^{-1}$. Errors associated with this diagram may be larger than $\pm 5 \mathrm{~km} \mathrm{~s}^{-1}$ as discussed in Sect. 5 .

Another aspect to be investigated is the analysis of evolving long-duration oscillatory phenomena. The four-dimensional power maps produced during wavelet analysis will be studied for signs of evolution and/or power variability.

Acknowledgements. This work was supported by the UK Particle Physics and Astronomy Research Council. D.B.J. is supported by a Northern Ireland Department for Employment and Learning studentship. DBJ additionally thanks NASA Goddard Space Flight Center for a CAST studentship - in particular Doug Rabin and Roger Thomas deserve special thanks for their endless help, support and scientific input. FPK is grateful to AWE Aldermaston for the award of a William Penney Fellowship. Observations were obtained at the National Solar Observatory, operated by the Association of Universities for Research in Astronomy, Inc. (AURA), under cooperative agreement with the National Science Foundation. We are grateful to the anonymous referee for pointing out highly informative references which aided in the interpretation of our results. We would also like to thank Kevin Reardon and Gianna Cauzzi for informal discussions related to the interpretation of the findings presented in this paper. Finally we would like to thank the technical staff at the DST for perseverance in the face of atrocious weather conditions. Wavelet software was provided by $\mathrm{C}$. Torrence and G. P. Compo ${ }^{1}$.

\section{References}

Andić, A. 2007, Sol. Phys., accepted

Athay, R. G., \& White, O. R. 1978, ApJ, 226, 1135

Athay, R. G., \& White, O. R. 1979a, ApJS, 39, 333

Athay, R. G., \& White, O. R. 1979b, ApJ, 229, 1147
Banerjee, D., O'Shea, E., Doyle, J. G., \& Goossens, M. 2001, A\&A, 371, 1137 Beckers, J. M., Dickson, L., \& Joyce, R. S. 1975, A Fully Tunable Lyot-Öhman Filter (AFCRL-TR-75-0090; Bedford: AFCRL)

Bellot Rubio, L. R., Balthasar, H., Collados, M., \& Schlichenmaier, R. 2003, A\&A, 403, 47

Biermann, L. 1948, Zeitschrift fur Astrophysics, 25, 161

Bloomfield, D. S., McAteer, R. T. J., Mathioudakis, M., Williams, D. R., \& Keenan, F. P. 2004, ApJ, 604, 936

Bloomfield, D. S., Solanki, S. K., Lagg, A., Borrero, J. M., \& Cally, P. S. 2007, A\&A, accepted

Brisken, W. F., \& Zirin, H. 1997, ApJ, 478, 814

Brynildsen, N., Maltby, P., Fredvik, T., \& Kjeldseth-Moe, O. 2002, ESA-SP, 506 de Boer, C. R. 1995, A\&AS, 114, 387

Deubner, F.-L. 1975, A\&A, 44, 371

Ding, M. D., Qiu, J., Wang, H., \& Goode, P. R. 2001, ApJ, 552, 340

Fossum, A., \& Carlsson, M. 2005a, Nature, 435, 919

Fossum, A., \& Carlsson, M. 2005b, ApJ, 625, 556

Giovanelli, R. G. 1972, Sol. Phys., 27, 71

Heinzel, P. A. 1991, Sol. Phys., 65

Hollweg, J. V. 1981, Sol. Phys., 70, 25

Ireland, J., Walsh, R. W., Harrison, R. A., \& Priest, E. R. 1999, A\&A, 347, 355

Jefferies, S. M., McIntosh, S. W., Armstrong, J. D., et al. 2006, ApJ, 648, 151

Kobanov, N. I., Kolobov, D. Y., \& Makarchik, D. V. 2006, Sol. Phys., 238, 231

Leenaarts, J., Rutten, R. J., Sütterlin, P., Carlsson, M., \& Uitenbroek, H. 2006, A\&A, 449, 1209

Leighton, R. B. 1960, IAUS, 12, 321

Lites, B. W. 1992, Proceedings of the NATO Advanced Research Workshop on the Theory of Sunspots, 261

McAteer, R. T. J., Gallagher, P. T., Williams, D. R., et al. 2003, ApJ, 587, 806

McAteer, R. T. J., Gallagher, P. T., Bloomfield, D. S., et al. 2004, ApJ, 602, 436

Makarchik, D. V., \& Kobanov, N. I. 2001, IAU Symp., 203

Marcu, A., \& Ballai, I. 2005, PADEU, 15, 103

Mathioudakis, M., Seiradakis, J. H., Williams, D. R., et al. 2003, A\&A, 403, 1101

Phillips, K. J. H. 2000, Sol. Phys., 193, 259

Rimmele, T. R. 2004, ApJ, 604, 906

St. John, C. E. 1913, ApJ, 37, 322

Schlichenmaier, R., \& Solanki, S. K. 2003, A\&A, 411, 257

Schlichenmaier, R., Jahn, K., \& Schmidt, H. U. 1998, A\&A, 337, 897

Schlichenmaier, R., Müller, D. A. N., Steiner, O., \& Stix, M. 2002, A\&A, 381, 77

Schwarzschild, M. 1948, ApJ, 107, 1

Solanki, S. K. 2003, A\&AR, 11, 153

Stein, R. F., \& Leibacher, J. 1974, ARA\&A, 12, 407

Sütterlin, P., Hammerschlag, R. H., Bettonvil, F. C. M., et al. 2001, ASP Conf. Ser., 236

Torrence, C., \& Compo, G. P. 1998, Bull. Amer. Meteor. Soc., 79, 61

Uitenbroek, H., \& Tritschler, A. 2006, ApJ, 639, 525

van Noort, M. J., \& Rouppe van der Voort, L. H. M. 2006, ApJ, 648, L67

von der Lühe, O. 1993, Sol. Phys., 268, 374

Wang, H., Qiu, J., Denker, C., et al. 2005, ApJ, 542, 1080

Wedemeyer-Böhm, S., Steiner, O., Bruls, J., \& Rammacher, W. 2007, ASP Conf. Ser., Coimbra Solar Physics Meeting

Weigelt, G., \& Wirnitzer, B. 1983, Optics Lett., 8, 389

White, O. R., \& Athay, R. G. 1979a, ApJS, 39, 317

White, O. R., \& Athay, R. G. 1979b, ApJS, 39, 347

Williams, D. R., Phillips, K. J. H., Rudawy, P., et al. 2001, MNRAS, 326, 428

Williams, D. R., Mathioudakis, M., Gallagher, P. T., et al. 2002, MNRAS, 336, 747

Zirin, H., \& Stein, A. 1972, ApJ, 178, 85

1 Wavelet software is available at http://paos. colorado.edu/research/wavelets/ 